\title{
Fabrication, testing, and performance of a variable-focus $x-$ ray compound lens
}

\author{
A. Khounsary ${ }^{*}$, S. D. Shastri ${ }^{a}$, A. Mashayekhi ${ }^{a}$, A. Macrander ${ }^{a}$, R. Smither ${ }^{a}$, F. F. Kraft ${ }^{b}$ \\ ${ }^{a}$ Advanced Photon Source, Argonne National Laboratory, Argonne, IL 60439, USA \\ ${ }^{b}$ Mechanical Engineering Department, Ohio University, Athens, OH 45701, USA
}

\begin{abstract}
ABSTACT
Design, fabrication, testing, and performance of an x-ray lens assembly are described. The assembly consists of a number of precisely stacked and aligned parts, each of which is a section of an extruded aluminum piece having 16 parabolic cavities. The wall thickness between adjacent cavities is $0.2 \mathrm{~mm}$. By stacking a number of long, extruded parts and cutting the assembly diagonally, a variable-focus lens system is devised. Moving the lens horizontally allows the incident beam to pass through fewer or more cavities focusing the emerging beam at any desired distance from the lens.

The variable focus aluminum lens has been used at the Advanced Photon Source to collimate a monochromatic, $8 \mathrm{keV}$ undulator beam. Results indicate collimation consistent with theoretical expectations.
\end{abstract}

Keywords: x-ray optics, focusing, compound x-ray lens, variable focus lens, aluminum extrusion

\section{INTRODUCTION}

Focusing or collimating diverging $\mathrm{x}$-ray beams produced by synchrotron sources or other $\mathrm{x}$-ray sources is important for a range of applications. Mirrors, crystals, zone plates, and capillaries have been suggested and used to focus x-rays. Focusing x-rays by refraction, proposed a few decades ago and presumed ineffective, has recently been implemented through the use of a compound x-ray lenses (CXL). ${ }^{1}$

The real part of the index of refraction for x-rays is close to unity, and as such-and unlike at visible wavelengthsrefraction is not an effective means to bend $\mathrm{x}$-rays. It takes quite a number of refractions to effect an appreciable bending of an $\mathrm{x}$-ray beam. A compound $\mathrm{x}$-ray lens system used to accomplish this consists of $10 \mathrm{~s}$ to $100 \mathrm{~s}$ of aligned lenses. Because the index of refraction for x-rays is slightly smaller than unity, these lenses are concave. Figure 1 depicts focusing by refraction.

For a lens system consisting of $\mathrm{N}$ concave lenses of radius of curvature $\mathrm{R}$, the focal length is simply given by:

$$
F=\frac{R}{2 N \square},
$$

where $\square$ is the real index of refraction decrement. The index of refraction is given by $n=1 \square \square+i \square$. From the relationship above, it can be deduced that a CXL positioned at $30 \mathrm{~m}$ from the source to collimate a $10-\mathrm{keV} \mathrm{x}$-ray beam, should have five 1-mm-radius cavities. To focus the same $2 \mathrm{~m}$ downstream, it should have an additional 74 lenses.

The main drawback of the CXL is its substantial absorption of x-rays. Thus, the use of this type of a lens is justifiable if

Corresponding author: Ali Khounsary, 630-252-3384, AMK@APS.ANL.GOV. This work was supported by the U.S. Department of Energy , Office of Science, Office of Basic Energy Sciences, under Contract No. W-31-109-Eng-38. 
the gain by focusing more than offsets the loss due to absorption. The lens materials suitable for focusing $\mathrm{x}$-rays in the 5-100 keV range are the low-atomic-number elements, such as $\mathrm{Li}, \mathrm{Be}, \mathrm{B}, \mathrm{C}, \mathrm{Al}$, Si, etc., and their compounds. For lowto-moderate-energy x-rays $(5-20 \mathrm{keV}), \mathrm{Li}$ and Be are most suitable because of their low absorption. For higher energies, $\mathrm{Al}$ and other elements or compounds can be used.

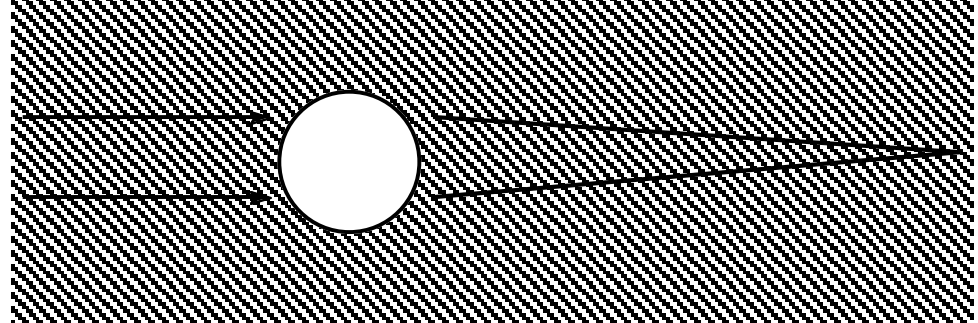

Fig. 1: Focusing $\mathrm{x}$-rays can be accomplished by using concave lenses. In this figure, a substrate is shown consisting of two one-sided concave lenses configured by drilling a hole. Focal lengths are typically in the $10 \mathrm{~s}-100$ s of meters, which is why a large number of aligned lenses are used to obtain short useful focal lengths (under a meter).

The other challenge in the development of CXLs is in fabrication. A suitable lens system is one that is relatively easy and economical to manufacture, provides thin walls between adjacent cavities (to reduced absorption), is robust, requires no or little alignment of individual lenses, and has relatively smooth walls.

A variety of schemes to fabricate CXLs have been suggested. These range from routine machining processes, such as milling or drilling, to more specialized processes, such as electrical-discharge machining (EDM), molding, stamping, etching, casting, electroplating, etc.

If one-dimensional focusing is sought, then a substrate with a number of aligned cylindrical holes drilled into it may be used. If two-dimensional focusing is desired, then spherical cavities may be configured. To reduce optical aberrations, the holes and cavities should have parabolic and paraboloidal shapers, respectively.

The earliest CXLs were aluminum lenses developed at the European Synchrotron Radiation Facility (ESRF) by drilling a set of aligned holes into an aluminum block with a thin wall between adjacent holes. This was used for onedimensional focusing. The Advanced Photon Source (APS) developed the first two-dimensional focusing lens in Be by configuring one hundred 1-mm-radius hemispheres into a beryllium blocks. ${ }^{2}$ Cutting the block into two and matching and aligning the hemispheres made a lens assembly consisting of fifty spherical cavities.

In this paper, a CXL system for high-energy x-rays developed by extrusion of aluminum is described. This is a onedimensional focusing device, but, in theory, two sets of such systems can be set normal to each other to focus in both directions (at the expense of doubling absorption). Because of its high absorption of low-energy photons, an aluminum lens is deemed suitable for energies above $30 \mathrm{keV}$ and preferably above $60 \mathrm{keV}$.

\section{EXTRUDED ALUMINUM LENS}

The extrusion technique used here is more appropriately known as hot microextrusion. In hot extrusion of hollow or tubular profiles, a die is used that has openings corresponding to the negative of the part to be made. A hollow extrusion die is one in which the mandrel, which forms the inner surfaces of the profile, is an integral part of the die assembly and is supported in place by a bridge. Allowance for thermal shrinkage and other effects are made in the die design. By pushing heated soft aluminum into the die, soft aluminum flows around the bridge supporting the mandrel and into the openings in the die. The open sections metallurgically join prior to exiting the die assembly to make the desired part. After cooling, the long, extruded section or lens is coiled or cut into the desired length for shipping. 


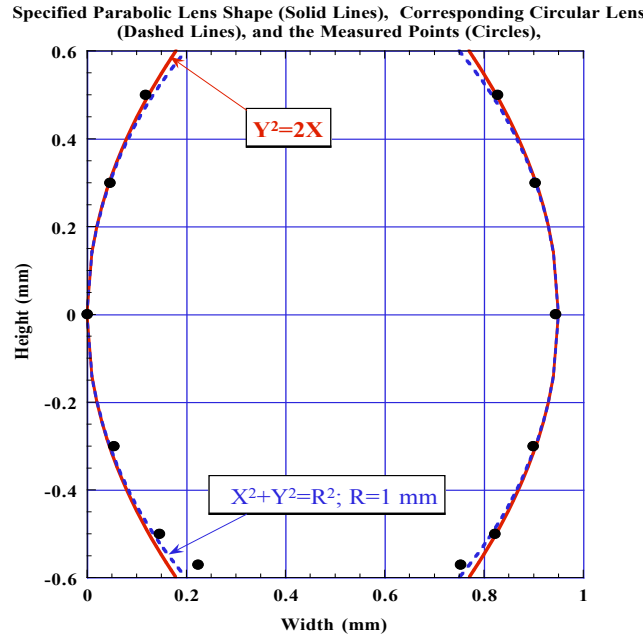

Fig. 2: Measured cross-sectional profile of a typical lens, and comparison with parabolic and circular cross sections.
The first generation of the lenses produced and reported here had a cross-section of $2 \mathrm{~mm} \times 20 \mathrm{~mm}$, with 16 parabolic cavities. The on-axis radius of curvature is $1 \mathrm{~mm}$, and the thickness of the wall between adjacent cavities is about $0.2 \mathrm{~mm}$. Fig. 2 shows a typical cross section of a single lens. The desired parabolic profile, as well as the circular approximation to it, is shown. Variation between the latter two profiles is more evident away from optical axis. Exhaustive evaluation of the lens cross sections were not performed, but Fig. 2 seems typical.

To make a lens assembly, a sufficient number of the extruded parts are cut into shorter lengths (via EDM) and laid side-by-side to make the assembly with any desired number of cavities (see Fig. 3). The pieces are sandwiched between two flat plates to align and maintain alignment. This section is then inserted into the opening of a u-shaped aluminum holder and held there by a number of set screws.

The assembly is then diagonally cut, and as shown in Fig. 3, a variable focus devise is produced: by moving the assembly horizontally, the incident beam passes through fewer or more cavities focusing the incident beam vertically at different focal spots. As seen, one section of the assembly has a fixed number of lenses, in this case 70, as it was deemed unnecessary to have continuous variation of the number of lenses down to zero.
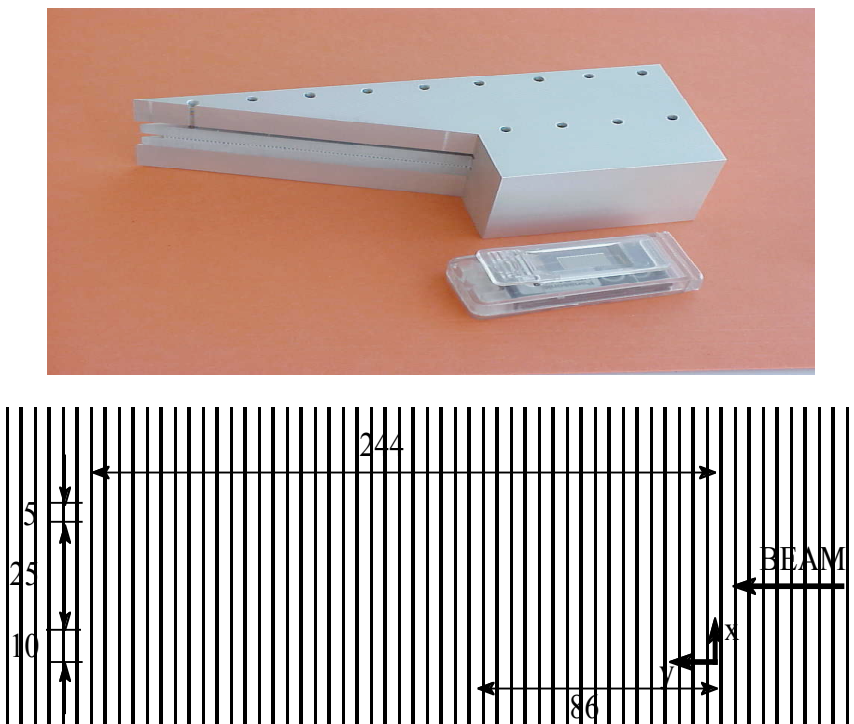

Fig. 3: Top picture is a prototype; aluminum-extruded variable-focus $\mathrm{x}$ ray lens assembly. The assembly consists of a U-shaped aluminum block in which the two flat plates sandwiching the extruded pieces are laid side by side between them and inserted and held in position by several set screws. The assembly is then cut by EDM to provide the sloped section seen, giving the assembly its variable focus ability. The drawing in the bottom shows the top view of the lens assembly tested and its dimensions in $\mathrm{mm}$. During the test, the lens assembly is moved horizontally in the $\mathrm{x}$-direction to allow the beam to transverse fewer or more cavities. 
Vertical Beam Size Downstream of the Variable Focus Lens

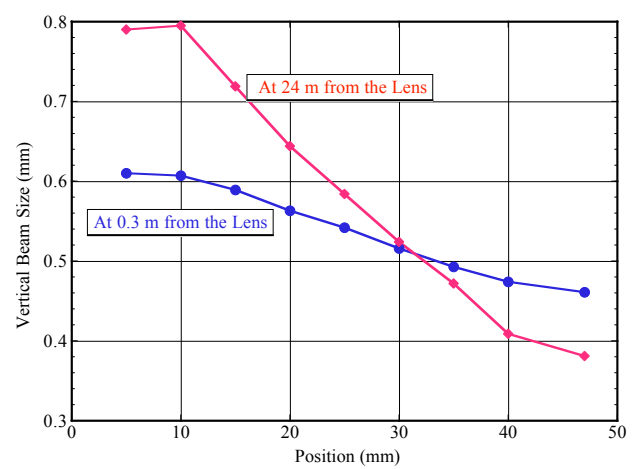

Vertical Beam Size Downstream of the Variable Focus Lens

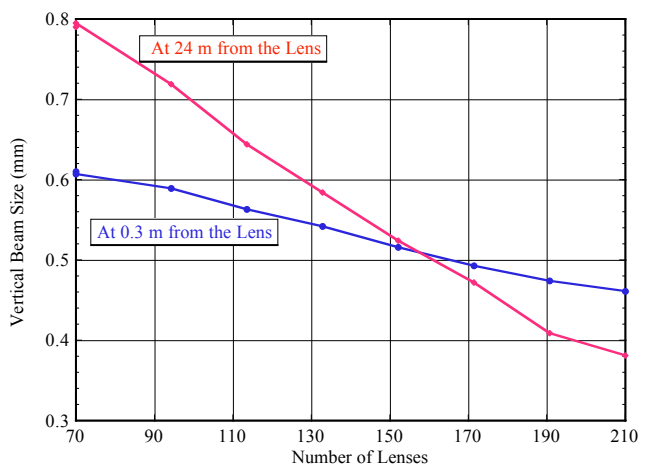

Fig. 5: Vertical FWHM of the x-ray beam at $0.3 \mathrm{~m}$ and $24 \mathrm{~m}$ downstream of the lens assembly as a function of the position (left) or the corresponding number of lenses through which the beam passes. Collimation is achieved when the beam has the same size at both locations. This occurs in this case at $x=32 \mathrm{~mm}$ corresponding to 157 lenses. The changes in the slope of the curves at the two ends are the result of the way the lens assembly is cut (see Fig. 3).

As seen in Fig. 5, collimation occurs at about $\mathrm{x}=32 \mathrm{~mm}$ corresponding to about 157 lenses. With that many lenses, the beam size immediately $(30 \mathrm{~mm})$ downstream of the lens is the same as that $24 \mathrm{~m}$ from the lens. The observed reduction in the beam size immediately downstream of the lens is purely due to the absorption: because of the curved shape of the lenses, the beam goes through a varying thickness of aluminum, and it is attenuated least at the center and most on the periphery. The flat parts of the curves in right side of the same figure correspond to the part of the lens assembly that is not sloped and has a fixed number (70) of lenses.

The real part of the index of refraction for aluminum at $81 \mathrm{keV}$ is $\square=8.23 \mathrm{E}-8$. As such, the number of lenses to collimate the beam from the source at $35 \mathrm{~m}$ is:

$$
N=\frac{R}{2 F \square}=\frac{1 \times 10^{\square 3}(\mathrm{~m})}{2[35(\mathrm{~m})]\left[8.23 \times 10^{\square 8}\right]}=174 .
$$

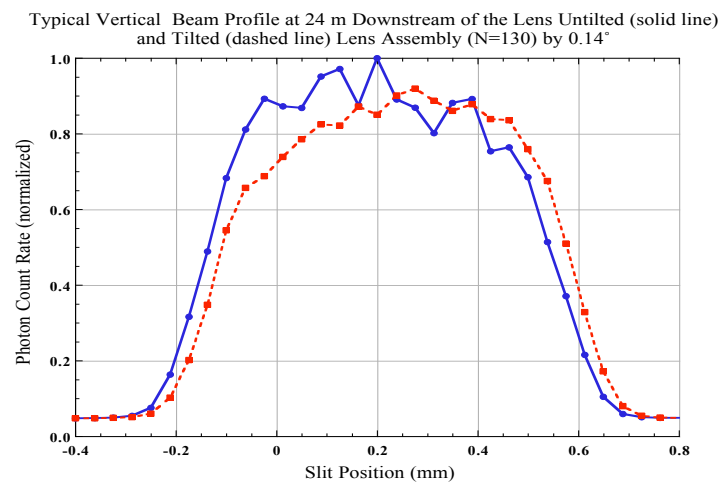

Fig. 6: The vertical profile of the x-ray beam emerging from the x-ray lens assembly. The variations are thought to be due to surface features in the lenses produced during extrusion. Tilting the lens vertically by $0.14^{\circ}$ smoothes but does not eliminate the observed features.
This value is within $10 \%$ of the measured value and is well within the error bar for this type of experiments because of uncertainty in lens radius, number of lenses traversed, index of refraction of the metal (due to impurities), etc.

A typical vertical profile of the $\mathrm{x}$-ray beam emerging from the lens system is shown Fig. 6. The observed intensity variations are thought to be due to imperfections in the lenses or their alignment. No provision for alignment other than sandwiching the lenses pieces between two flat plates were made. Figure 6 also shows that, by tilting the lens assembly by a fraction of degree vertically, the observed variations remain but are shifted. These features are not deemed detrimental for the intended application, however. 


\section{USING THE LENS ASSEMBLY FOR BEAM COLLIMATION}

One of the reasons for developing this lens assembly was to collimate the beam, thereby increasing the $\mathrm{x}$-ray throughput of subsequently placed narrow-angular-acceptance optics. ${ }^{4}$ In one case, a pair of flat $\mathrm{Si}(111)$ crystals in a dispersive arrangement is positioned immediately downstream of the lens assembly (not shown in Fig 4). The purpose is to further monochromatize the $81 \mathrm{keV}$ beam off the bent Laue pre-monochromator from an energy resolution of $120 \mathrm{eV}$ to $7 \mathrm{eV}$, as required for some applications. The Darwin width (i.e., the narrow angular acceptance range about the Bragg angle within which diffraction occurs) of the second set of monochromator crystals is a few microradians, about 10 times smaller than the divergence exiting the Laue monochromator. This means that, uncollimated, much of the radiation will not propagate through the high-resolution system. Collimating the beam can increase the throughput if the absorption in the lens is relatively small. Calculations show that, with 157 lenses and an average lens thickness of $0.22 \mathrm{~mm}$, only about $12 \%$ of the x-ray beam is transmitted $(=\exp (-\mu \mathrm{z}) \sim \exp (-0.054 \times 157 \times 0.22)=15 \%$. Yet with this huge $85 \%$ absorption, the throughput of the analyzer crystals only modestly deteriorated, by only between 10 to $20 \%$, meaning that, were it not for this absorption, the collimating lens could increase the system throughput by more than a factor of 6 . Reducing the average wall thickness to $0.1 \mathrm{~mm}$ - in the second generation of lenses, will increase transmission to $43 \%$ and more than double the system throughput, making the use of this lens well worthwhile.

\section{CONCLUSIONS}

The fabrication of an extruded aluminum compound x-ray lens assembly with a parabolic profile is described. The lens system design has a continuous variable-focus feature giving it on-line operational versatility. The assembly can be cooled and, as such, can be used on monochromatic as well as white beam beamlines. Collimation was demonstrated on an undulator beamline at the APS, and the results are in good agreement with theory. Thus production of x-ray lenses by aluminum extrusion is possible, and the extrusion process used has sufficient precision to render parts that have acceptable profile, figure and finish. The process is very economical. The next-generation x-ray lenses, having a wall thickness of $0.1 \mathrm{~mm}$, have been fabricated and will be tested shortly for collimation and focusing.

\section{ACKNOWLEDGMENT}

The authors wish to thank Mr. Kevin Cole, Joe McDougall, and Brazeway Inc. for their help in manufacturing of the extruded parts. This work was supported by the U.S. Department of Energy, Office of Science, Office of Basic Energy Sciences, under Contract No. W-31-109-Eng-38.

\section{REFERENCES}

\footnotetext{
${ }^{1}$ Snigirev, A., V. Kohn, I. Snigireva, and B. Lengeler, “A compound refractive lens for focusing high-energy x-rays,” Nature 384, 4951, 1996.

${ }^{2}$ Smither, R, A. Khounsary, D. Roa, P. Fernandez, and D. Haeffner, "Refractive lenses using hollow spheres in beryllium and aluminum substrates," Presented at SRI Conf. In Berlin, Germany, 2002.

${ }^{3}$ Shastri, S. D., K. Fezzaa, A. Mashayekhi, W.-K. Lee, P.B. Fernandez, and P. L. Lee, "Cryogenically cooled bent double-Laue monochromator for high-energy x-rays (50-200k keV)," J. Synchrotron Rad. 9, 317-322, 2002.

${ }^{4}$ Baron, A. Q. R., Y. Kuhmura, Y. Oshishi, and T. Ishikawa, Appl. Phys. Lett. 74, 1492-1494, 1999.
} 